\title{
COGNITIVE TEST ANXIETY SCALE: PROPRIEDADES PSICOMÉTRICAS NO CONTEXTO BRASILEIRO*
}

\author{
COGNITIVE TEST ANXIETY SCALE: \\ PSYCHOMETRIC PROPERTIES FOR THE BRAZILIAN CONTEXT
}

Recibido: 25 de diciembre de 2019 | Aceptado: 29 de septiembre de 2020

EMERSON DIÓGENES DE MEDEIROS 1, PAULO GREGÓRIO NASCIMENTO DA SILVA 2,

PALOMA CAVALCANTE BEZERRA DE MEDEIROS 3, GISLY MACEDO DE SOUSA 4, CAROLINE CABRAL NUNES 5,

PATRÍCIA NUNES DA FONSÊCA 6, ERNANDES BARBOSA GOMES 7

1., 3. UNIVERSIDADE FEDERAL DO Delta do PARNaíBA, Parnaíba-PI, Brasil

2., 6., UNIVERSIDADE FEDERAL DA PARAíBA, João Pessoa-PB, Brasil

4., 5., 7. UNIVERSIDADE FEDERAL DO PIAUÍ, Parnaíba-PI, Brasil

\begin{abstract}
BACKGROUND: Cognitive anxiety refers to excessive worries before, during and after a test, interfering on performance and causing academic failure, and is positively associated with neuroticism and depression. Objective: The objective was to gather evidence of psychometric properties of the Cognitive Test Anxiety Scale (CTAS) in Brazil. METHOD: Two studies were conducted: For Study 1, 210 university students from the city of Parnaíba, Piauí, Brazil (Mean age $=21.7 ;$ SD $=3.73$ ) participated, most of them studying Psychology $(60 \%)$ and women (58.6\%). They answered CTAS and demographic questions. RESULTS: An exploratory factor analysis suggested a one-dimensional solution that explained $46.94 \%$ of the total variance of the items. Reliability was evaluated by Cronbach's alpha $(\alpha)$ and McDonald's omega $(\omega)$ indicators equal to .92. In Study 2, 274 university students from different Brazilian regions participated (Mean age $=21.7 ; S D=3.73$ ), most of the state of Piaui (60\%), studying Psychology (60.4\%). A confirmatory factor analysis indicated adequate indicators $(\mathrm{CFI}=.96 ; \mathrm{TLI}=.95 ; \mathrm{RMSEA}=.06)$. The reliability was satisfactory: $(\alpha)$ and $(\omega)$ equal to .94 . CONCLUSIONS: Psychometric evidence was found for Brazil, and CTAS may be useful for those interested in investigating the theme and its correlates.
\end{abstract}

KEY WORDS: Test Anxiety, students, validity, factorial analysis.

\section{CÓMO CITAR / HOW TO CITE}

De Medeiros, E. D., Da Silva, P. G. N., De Medeiros, P. C. B., De Sousa, G. M., Nunes, C. C. Da Fonsêca, P. N., \& Gomes, E. B. (2020). Cognitive test anxiety scale: Propriedades psicométricas no contexto brasileiro. Salud \& Sociedad, 11, e3923. https://doi.org/10.22199/issn.0718-7475-2020-0004

\footnotetext{
* 0 presente artigo contou com apoio do CNPq e CAPES, por meio de financiamento projeto de pesquisa do CNPq ao primeiro autor e bol sa de produtividade a sexta autora, além de bolsa de Doutorado (CAPES) a segundo autor. Aproveitamos para demonstrar nossa gratidão a estas instituições. *Toda correspondência deverá ser encaminhada à Emerson Diógenes de Medeir os, telefone: (86) 999340260. Email: emersondiogenes@gmail.com; Univer sidade Federal do Piauí. Bairro: Nossa Senhora de Fátima - Parnaíba - PI. CEP: 64202 -020. Campus de Parnaíba - Departamento de Psicologia. 1. E-mail: emersondiogenes@gmail.com, 0RCID: https://orcid.org/0000-0002-1407-3433; 2. E-mail: silvapgn@gmail.com,0RCID: https://orcid.org/0000-0002-2878-309X;3.E-mail:palomacbmedeiros@gmail.com,0RCID: https://orcid.org/0000-0002-5868-8333. 4. E-mail: gislymacedo@gmail.com, ORCID: https://orcid.org/0000-0003-2324-6807; 5. E-mail: carolcabral_n@hotmail.com, 0RCID: https://orcid.org/0000-0001-9008-0144; 6. E-mail: pnfonseca.ufpb@gmail.com, 0RCID: https://orcid.org/0000-0002-6322-6336; 7. E-mail: ernandesb.gomes@gmail.com,0RCID: https://orcid.org/0000-0002-6647-1281
} 
$O$ contexto acadêmico pode representar um conjunto de situações estressoras devido ao fato de que este significa um novo ambiente para os estudantes recém-chegados do ensino médio. Além das mudanças na metodologia de ensino, com as quais os estudantes precisam aprender a lidar com a falta de controle sobre esse novo cenário e as novas formas de avaliações (Piemontesi \& Heredia, 2009). Nesta conjuntura, podem emergir acontecimentos acadêmicos que impactam negativamente 0 desempenho estudantil, ocasiona sobrecarga e ansiedade acadêmica (Thomas et al., 2017).

Dito isto, tem sido de particular relevância considerar a ansiedade vivenciada em situações avaliativas, uma vez que, em contexto universitário é possível verificar que cerca de 16,6\% estudantes de graduação apresentam níveis mais altos de ansiedade em situações avaliativas, com mulheres manifestando graus significativamente maiores em comparação com homens (Ne'Eman-Haviv \& Bonny-Noach, 2019), além de está relacionado positivamente com o neuroticismo e a depressão (Cassady et al., 2019).

Esta é uma característica situacional, é comumente referida como ansiedade frente a exames (AE) ou ansiedade de avaliação (Thomas et al., 2017), que envolve a preocupação com possíveis resultados negativos, ocorrendo, antes, durante e lou depois de um evento avaliativo (SánchezRosas \& Furlan, 2017). É uma resposta antecipatória, em situação que o indivíduo considera potencialmente aversiva, que visa a própria preservação, evitando e controlando eventuais problemas que possam surgir (Furlan et al., 2015).

$O$ estudo pioneiro sobre ansiedade frente a exames (AE), foi proposto por Sarason e Mandler (1952), que definiram o construto como uma resposta a realização de tarefas intelectuais que são consideradas ameaçadoras, tais como situações avaliativas, no qual o aumento da ansiedade ocasionará um pior desempenho ao indivíduo, sendo avaliado de forma global, ou seja, unidimensional. Entretanto, desde o final da década de 1960, especificamente, a partir da pesquisa de Liebert e Morris (1967), começou-se a considerar a $A E$ de forma mais ampla, por uma perspectiva bidimensional, que tradicionalmente foram denominadas de emoção, que é referente a reações autonômicas que tendem a ocorrer sob 0 estresse ocasionado pelo exame, além da preocupação, entendida como qualquer expressão cognitiva de preocupação com 0 próprio desempenho.

Ao longo dos anos as pesquisas tem sido consistentes para estes dois domínios da ansiedade, evidenciando, por exemplo, que índices elevados de emoção, estão relacionados a fatores fisiológicos específicos, tais como aumento na frequência cardíaca, transpiração e dores de cabeça (Sarason, 1984), ao passo que, a preocupação frente a avaliações incluem características como, maior ameaça percebida, processamento cognitivo inadequados de habilidades de organização e estratégias de estudo, vulnerabilidade à interferência cognitiva (isto é, distração) durante os períodos de estudos e provas, além de perspectivas motivacionais que promovem comportamentos de evitação da tarefa, estratégias de enfrentamento desengajadas e aceitação de falhas (Cassady \& Finch, 2015).

Especificamente, nos últimos anos, a dimensão preocupação também tem sido entendida como como ansiedade cognitiva frente a exames/avaliações (Cassady \& Johnson, 2002; Cassady, 2010), referindo-se a reações cognitivas em situações avaliativas, ou diálogo interno sobre tais situações, antes, durante e depois as tarefas avaliativas (Furlan et al., 2009), envolvendo algumas características, tais como comparação do próprio desempenho com os pares, considerações sobre as consequências do fracasso, baixos níveis de confiança no próprio desempenho, preocupação excessiva, possi- 
bilidade de causar tristeza aos seus pais, sensação de despreparo, e perda de autoestima (Cassady \& Johnson, 2002).

Embora esses elementos estejam fortemente correlacionados, tem se admitido que estes devem ser considerados como construtos teoricamente distintos, mas empiricamente relacionados (Putwain et al., 2015), havendo um consenso de que os indicadores fisiológicos de emoção, são considerados mais gerais, e que a ansiedade cognitiva frente a exames (preocupação) está mais diretamente relacionada aos níveis de desempenho nas avaliações (Cassady \& Finch, 2015), Assim, ressalta-se que a presente pesquisa se foca no componente cognitivo da $A E$ (preocupação), por ser 0 componete mais importante na explicação do desempenho acadêmico (Cassady \& Finch, 2015). Entende-se que quanto maior a preocupação (ansiedade cognitiva), menor será o foco e orientação na execução das tarefas, ocasionando um menor dessempenho acadêmico (Putwain et al., 2015).

Por exemplo, o estudo realizado por Cassady (2004) com 124 universtários da Midwestern United States University nos Estados Unidos. Os pesquisadores observaram que os alunos com alta ansiedade cognitiva classificaram as avaliações como mais ameaçadoras, apresentando menor capacidade de estudo e pior desempenho. Ademais, verificou-se que a ansiedade cognitiva está relacionada negativamente com percepções e engajamentos em comportamentos que estão associados a eventuais decréscimos de desempenho em todas as fases do ciclo aprendizagem (Cassady, 2004). Especificamente, na fase de reflexão sobre a avaliação, os alunos que se sentem desamparados e tendem a experimentar níveis mais elevados de ansiedade cognitiva.

Nessa direção, Cassady et al. (2004) verificaram diferenças de percepção do exame em universitárias do Kuwait e dos Estados Unidos. O primeiro grupo apre- sentou uma menor preocupação sobre as potenciais consequências negativas sobre 0 mal desempenho, além de uma relação negativa entre as habilidades do estudo e a ansiedade no momento da avaliação. Ao passo que mulheres americanas percebiam as avaliações como mais ameaçadoras

Dado o exposto, parece evidente que 0 componente o cognitivo é importante na explicação da $A E$, podendo influenciar 0 desempenho acadêmico. Devido a isto, Cassady e Johnson (2002) elaboraram a Cognitive Test Anxiety Scale - CTAS, destinada a mensurar os indicadores cognitivos da ansiedade frente a exames. 0 foco da CTAS é sobre a tendência a apresentar pensamentos intrusivos pensamento e de comparação com os pares no decorrer dos períodos de preparação e/ou durante a execução e preparação para a avaliação, ocasionando déficits de atenção no aluno (Furlan et al., 2009). Especificamente a medida em questão foca-se na preocupação, uma vez que medidas elaboradas previamente, abarcavam itens que refletiam processos cognitivos para além deste componente, tais como a interferência, às crenças autodestrutivas e pensamentos que não estavam relacionados à tarefa (Furlan et al., 2011).

Para tanto, foram considerados aspectos da preocupação referentes aos modelos de Liebert e Morris (1967) e de Sarason (1984). Assim, os itens foram elaborados a fim de representar diferentes aspectos do fenômeno (Cassady \& Johnson, 2002), citados previamente. A versão preliminar da CTAS proposta por Cassady e Johson (2002), era composta inicialmente por 44 itens, respondidos em uma escala do tipo Likert de 4 pontos, variando de 1 "nada frequente em mim" a 4 "muito frequente em mim". A pesquisa empírica com 400 universitários, por meio de análise fatorial, que possibilitou observar uma solução composta por um fator geral, que reuniu 27 itens, com adequada consistência interna (precisão; $\alpha=0,88$ ). Além disso, verificou-se relações positivas 
com procrastinação e o com emoção e relações negativas desempenho acadêmico. Pesquisas posteriores, com amostras de universitários corroboram a unidimensionalidade da CTAS (Baghaei \& Cassady, 2014; Cassady \& Finch, 2015; Furlan et al., 2009; Furlan et al., 2011).

Tomando como exemplo alguns desses estudos, pode-se citar a pesquisa de Furlan et al. (2009), que levaram a cabo uma pesquisa com 752 universitários, visando adaptar a medida para o contexto argentino. Por meio de dois estudos (exploratório e confirmatório), foi possível verificar indicadores meritórios de estrutura interna da medida, que foi reduzida para 16 itens em uma solução unifatorial, com adequado índice de precisão $(\alpha=0,88)$, que apresentou uma forte correlação com a versão original de 27 itens $(r=0,94, p<0,0001)$, indicando a possibilidade de avaliar o construto por uma medida abreviada, seguindo o critério da parcimônia. Ademais, ressalta-se, que 0 segundo fator foi excluído, pois os itens apresentaram interpretação problemática, que segundo os autores, foi devido a sua redação e codificação inversa, que caracterizava baixa ansiedade (Furlan et al., 2011).

Ademais, por meio das versões de 27 e 16 itens, testaram-se modelos alternativos (uni e bifatorial), por meio da análise fatorial confirmatória, corroborando a estrutura unidimensional. A estabilidade temporal da medida foi atestada por meio do teste-reteste (intervalo de 90 dias), verificando-se uma correlação forte $\quad(r=0,77, \quad p<0,01)$. Posteriormente, está solução unifatorial foi corroborada, por Furlan et al. (2011), que normatizaram a medida, estabelecendo pontos de cortes em função do sexo para 0 contexto argentino. Ademais, evidencia-se que está medida tem sido utilizada em diversas pesquisas transculturais: Argentina (Furlan et al., 2011; Sánchez-Rosas \& Furlan, 2017); Kuwait (Cassady et al., 2004); Estados Unidos (Cassady, 2004); Irã (Baghaei \& Cassady, 2014) e Turquia (Bozkurt, Ekitli, Thomas, \& Cassady, 2017).
Já Cassady e Finch (2014) conduziram uma pesquisa com 742 universitários americanos, na qual averiguaram a estrutura psicométrica para os 27 itens da CTAS, constataram uma estrutura bifatorial, na qual os itens com codificação inversa se agruparam, gerando um segundo fator, denominado confiança. Assim, foram testados modelos alternativos: (a) modelo composto pelos 27 itens originas, distribuídos em dois fatores; (b) os 27 itens saturando em um único fator e (c) Os 17 itens, oriundos da exclusão dos codificados inversamente. Os resultados sugeriram que versão reduzida com estrutura interna unifatorial era preferível. A exlusão dos demais itens ocorreram devido as ambiguidades ocasionadas pelos itens de codificação inversa, como já havia sido observado préviamente (Furlan et al., 2009), podendo sugerir que os itens com codificação inversa corrospodiam a um construto conceitualmente diferente.

Tendo em conta isto, argumenta-se que contar com um instrumento com um número reduzindo de itens, pode aumentar o escopo de pesquisa, minimizando a quantidade de tempo e esforço gasto pelos participantes, sem expandir o período de avaliação desnecessariamente (Furlan et al., 2011). Assim, considerando a versão proposta por Cassady \& Finch, (2014), Baghaei e Cassady (2014) levaram a cabo uma pesquisa com 297 universitários, adaptando a CTAS para o Irã, avaliando a medida por meio do modelo Rasch, que apontou uma estrutura unifatorial. Além disso, estimativa para o funcionamento diferencial do item (Differential Item Functioning, DIF), que demonstrou que os itens em função do sexo, evidenciando que, de maneira geral, mulheres apresentam níveis mais elevados de ansiedade.

Posteriomente, Bozkurt et al., (2017) adaptaram a medida para a Túrquia, contando com uma amostra de estudantes do ensino médio da cidade de Izmir. As análises fatoriais (exploratória e confirmatória), reforçaram a solução unifatorial, que 
reuniu 23 itens. Ademais, foram reunidas evidências de validade convergente, onde a CTAS apresentou correlações positivas e moderadas com o Text Anxiety Inventory (TAl; Spielberger, 1980), a saber: emoção $(r=0,54, \quad p<0,001)$ e preocupação $(r=0,57$, $p<0,001)$. Adicionalmente, por meio da técnica DIF, foi verificado a equivalência do funcionamento dos itens em função do sexo dos participantes. Entretanto, 0 Test $T$ demonstrou que mulheres apresentam níveis mais elevados de ansiedade quando comparadas com homens.

Em suma, entende-se que apesar existirem de diferentes versões da CTAS, oriundos do esforço de refinamento do instrumento e diferentes países, que possibilitaram refinar o instrumento, reunindo evidências que apontam sua unidimencionalidade, além de propocionar um melhor entendimento referente aos antecedentes e consequentes da ansiedade cognitiva em contextos avaliativos. Entretanto, considerando especificamente o cenário nacional, não foi encontrado nem uma pesquisa, evidenciando uma lacuna no Brasil. Portanto, como a CTAS é teoricamente fundamentada e apresenta propriedades psicométricas sólidas, ter uma versão em português brasileiro é importante, pois testar as propriedades psicométricas da CTAS no Brasil pode viabilizar a condução de estudos, que auxiliem na sistematização e melhor entendimento da temática.

Assim, o presente estudo tem como objetivo adaptar, reunindo evidências de estrutura interna da Cognitive Test Anxiety Scale para 0 português brasileiro, especificamente inclui avaliar a estrutura interna dos itens (validade fatorial e precisão) em universitários brasileiros. Para tanto, será considerada a versão proposta por Furlan et al. (2009), validada no contexto argentino e composta por 16 itens. Ressalta-se que optou-se por considerar a versão espanhola da CTAS devido as semelhanças do idioma espanhol com o português (em comparação com o idioma inglês), além disso deve-se ter em conta que a versão espanhola durante 0 processo de tradução realizado por Furlan e colaboradores, foi inspecionada e aprovada pelos autores da versão original da CTAS. Além disso, a versão composta por 16 itens, tem se apresentado mais parcimoniosa (Furlan et al., 2011). Assim, levou-se a cabo dois estudos empíricos distintos, a serem apresentados a seguir.

\section{MÉTODO}

\section{Participantes}

Contou-se com uma amostra total, não probabilística (de conveniência) de 415 universitários ( $M_{\text {idade }}=21,84$; amplitude 18 a 46 anos), de diferentes estados brasileiros. Estes eram em maioria do Piauí (94\%), do sexo feminino $(60,1 \%)$, oriundos de instituições públicas $(96,6 \%)$ e cursando Psicologia (34,9\%), Agronomia (14,7\%) e Biomedicina (3,1\%). A seguir, serão apresentados os perfis sociodemográficos dos participantes, que foram divididos em duas amostras distintas, que compuseram 0 estudo 1 (N1) e o estudo (N2).

N1 - Participaram 210 universitários de instituições públicas da cidade de Parnaíba, Piauí, Brasil ( $M_{\text {idade }}=21,7$; DP=3,73; amplitude 18 a 37 anos), a maioria cursando Psicologia (60\%) e mulheres (58,6\%). Estes foram recrutados de maneira acidental, não probabilística.

N2 - Participaram do estudo 205 universitários, selecionados de maneira acidental não-probabilística (por conveniência) de diferentes estados brasileiros, que tinham em média 21,76 anos de idade ( $D P=4,13$; amplitude 18 a 46 anos). Estes em maioria eram oriundos do estado do Piauí $(90,1 \%)$, de instituições públicas $(92,6 \%)$, mulheres $(61,2 \%)$ E cursando Psicologia (58\%).

\section{Instrumentos}

Os participantes responderam um livreto contento os seguintes instrumentos: 
Cognitive Test Anxiety Scale - (CTAS): elaborada Cassady e Johnson (2002), foi adaptada para o contexto Argentino por Furlan et al (2009). A adaptação argentina da escala ficou composta por 16 itens, que apresentaram boas qualidades psicométricas, versão que será considerada nesta oportunidade tendo em vista os procedimentos rigorosos e as análises estatísticas robustas na obtenção de seus índices psicométricos. Os itens (e.g. "item 03: Durante as avaliações eu penso muito nas consequências de uma reprovação") são respondidos em escala de cinco pontos tipo Likert, variando entre 1 "Nada frequente em mim" a 4 "Muito frequente em mim".

Questionário sociodemográfico. Os participantes responderam a um conjunto de perguntas, a exemplo de sexo, idade, estado civil e renda familiar, rendimento acadêmico, etc; que foram utilizadas com o objetivo de caracterizar a amostra.

\section{Procedimento}

Inicialmente, utilizando-se dos procedimentos recomendados por Borsa Damásio e Bandeira (2012), procedeu-se a tradução e adaptação da escala para o contexto brasileiro, dentre as quais deve-se enfatizar o método de tradução reversa (backtranslation). Para tanto, optou-se por por considerar no processo de tradução a versão espanhola da CTAS (Furlan et al., 2009), tendo em conta a semelhança do idioma espanhol com o português (em comparação com o idioma inglês), além disso a versão espanhola foi inspecionada de furlan e colaboradores foi inspecionada e aprovada pelo autor da versão original da CTAS. Assim, a escala foi traduzida para 0 português brasileiro, por dois tradutores independentes e em seguida retraduzida para o espanhol, por meio de traduções às cegas, visando verificar a equivalência dos itens das duas versões (português e espanhol). Ressalta-se que os tradutores estiveram atentos aos ajustes semânticos e idiomáticos, fazendo adequações e correções nos itens, quando necessário.
Posteriormente, a medida passou por um estudo piloto, 0 processo de validação semântica, que contou com a participação de 10 estudantes universitários, de ambos os sexos, estes dos primeiros e dos últimos períodos. Nesta oportunidade, foi verificado possíveis itens dos instrumentos (e.g. compreensão e deselegância), seguindo os procedimentos estabelecidos por Pasquali (2016). Superada esta etapa, tratou-se de aplicar os instrumentos. Nest oportunidade, aplicou-se os instrumentos de formas presencial, em ambiente de sala de aula, porém os estudantes respondiam de maneira individual, levando aproximadamente 20 minutos, em média, para finalizar a participação na pesquisa. Além da coleta em formato eletrônico, através do Google Docs. Aos que aceitaram colaborar, era, disponibilizado um link da pesquisa, além do mais, o link foi disponibilizado por meio das redes sociais (por exemplo, Instagran, Facebook e Twiter) e e-mails. Para tanto, contou-se a técnica de bola de neve para angariar participantes para a pesquisa.

Aos participantes, foi apresentado no Termos de Consentimento Livre e Esclarecido para que os participantes (alunos devidamente matriculados na instituição participante) pudessem assinalar e autorizar sua participação na pesquisa e responder aos instrumentos. Foi assegurado a todos 0 caráter anônimo e confidencial das respostas, além disso, era enfatizado 0 caráter voluntário da participação na pesquisa e que não traria nenhum prejuízo ou bônus aos participantes, e que poderiam desistir a qualquer momento sem ônus. Ademias, o presente estudo foi submetido e aprovado pelo o Comitê de Ética em Pesquisa do Centro de Ciências da Saúde (CEP/CCS) de uma instituição pública do nordeste brasileiro, com 0 CAAE: 89034618.5.0000.5188 e Parecer $n^{0}$ 2.731.766, estando de acordo com todos os preceitos legais previstos nas Resoluções 466/12 e 510/2016. 
Análise

Por meio do software Factor 9.2 (LorenzoSeva \& Ferrando, 2013) averiguou-se a dimensionalidade do CTAS, tendo em conta o método Hull Comparative Fit Index (CFI; Lorenzo-Seva, Timmerman, \& Kiers, 2011), através de uma análise fatorial exploratória categórica ULS (Unweighted Least Squares) e matriz de correlações policóricas. Ressalta-se que o método Hull configura-se como um dos melhores na estimação da dimensionalidade de um dado conjunto de itens (Lorenzo-Seva et al., 2011). Com o software $\mathrm{R}$ efetuaram-se análises descritivas para caracterizar a amostra. Utilizando-se do pacote Lavaan (Rossel, 2012) foi executada uma análise fatorial confirmatória (AFC) categórica (ordinal), com estimador Weighted Least Squares Mean and Variance-Adjusted (WLSMV; Muthén \& Muthén, 2014), o qual é implementado na matriz de correlações policóricas e considera a natureza ordinal dos dados. Utilizamos os seguintes indicadores para avaliar a adequação do modelo (Byrne, 2010; Tabachnick \& Fidell, 2013):

(1) Comparative Fit Index (CFI) - índice comparativo, frequentemente, valores a partir de 0,90 são referências de um modelo ajustado; (2) Tucker-Lewis Index (TLI) apresenta uma medida de parcimônia entre os índices do modelo proposto e do modelo nulo, varia de zero a um, com valores acima de 0,90 como aceitáveis; (3) Root-MeanSquare Error of Approximation (RMSEA) e seu intervalo de confiança de $90 \%$ (IC90\%) recomenda-se valores entre 0,05 e 0,08, admitindo-se até 0,10; (4) o Pclose é um indicador mais criterioso, testando a hipótese nula de RMSEA < 0,05, deste modo, recomenda-se 0 Pclose $>0,05$ como indicativo de modelo ajustado e (5) Root Mean Square Residual (RMSR) é a raiz quadrada matriz dos erros dividida pelo grau de liberdade, assumindo que o modelo é ajustado; Um RMSR < 0,08 indica um ajustamento adequado do modelo. 0 pacote rotina psych (Revelle, 2013), disponível no R, foi adotado para avaliar a confiabilidade, considerando os índices alfa de Cronbach e o ômega $(\omega)$ de McDonald. Para tanto, considerou-se a escala de resposta do tipo Likert como categorias ordenadas (HolgadoTello, Chacón-Moscoso et al., 2010; Lara, 2014).

\section{RESULTADOS}

\section{Estudo 1. Adaptação e Evidências de Validade e Precisão da Cognitive Test Anxiety Scale}

Inicialmente, realizamos uma análise fatorial exploratória, com a finalidade de conhecer a estrutura fatorial da matriz de correlações policóricas entre os 16 itens da CTAS. Por meio dos resultados do índice de KaiserMeyer-Olkin (KMO) $=0,93$ e o Teste de Esfericidade de Bartlett, $\chi^{2}(120)=1.656,5$; $p<0,001$, comprovou-se a pertinência de realizar a análise fatorial. 0 método Hull sugeriu uma solução unidimensional, após a verificação do índice de ajuste Global Fit Index $(\mathrm{GFI})=0,98$. O valor próprio (autovalor) foi de 7,51 e explicou $46,94 \%$ da variância total dos itens. As cargas fatoriais dos itens e a consistência interna da escala são apresentadas na Tabela 1.

Em suma, resultados apresentados na Tabela1 demonstram evidências de adequação de estrutura interna e precisão da CTAS, que apresentou uma estrutura unifatorial (como teorizado), reunindo 16 itens, com as saturações fatoriais variando entre 0,47 (item 15, "Nas avaliações não obtenho bons resultados") a 0,77 (item 07, "Durante as avaliações, eu fico tão nervoso que esqueço as coisas que realmente sei."). Ademais, a consistência interna (precisão), foi medida por meio do coeficiente alfa de Cronbach $(\alpha)$ com base em correlações policóricas e ômega de McDonald, ambas foram 0,92, sendo considerados meritórios.

Superada esta etapa, para que fosse assegurada as qualidades psicométricas da CTAS, reunimos evidências complementares de sua estrutura interna. Procedeu-se uma Análise Fatorial Confirmatória (AFC), por ser considerada uma técnica mais robusta. 
Ressalta-se que assim como no primeiro estudo, foi considerada a natureza categórica (ordinal) da medida para escolha do estimador a ser utilizado. Mais detalhes serão descritos a seguir.

TABELA 1.

Estrutura fatorial da Cognitive Test Anxiety Scale.

\begin{tabular}{|c|c|c|}
\hline Itens & $\begin{array}{c}\text { Carga } \\
\text { Fatorial }\end{array}$ & $h^{2}$ \\
\hline 7 - Durante as avaliações, eu fico tão nervoso que esqueço as coisas que realmente sei. & 0,77 & 0,60 \\
\hline 4 - Ao começar uma avaliação fico tão nervoso que muitas vezes não consigo pensar com clareza. & 0,75 & 0,57 \\
\hline 5 - Quando estou pressionado a responder uma avaliação, minha mente "dá um branco". & 0,73 & 0,54 \\
\hline 10 - Durante as avaliações tenho a sensação de que não estou indo bem. & 0,73 & 0,53 \\
\hline 6 - Durante as avaliações, muitas vezes eu tenho uma tendência a pensar que talvez não seja muito inteligente. & 0,72 & 0,52 \\
\hline 2 - Quando tenho que enfrentar avaliações finais sinto um bloqueio. & 0,71 & 0,51 \\
\hline 16 - Quando faço uma avaliação fico tão nervoso que cometo erros bobos. & 0,69 & 0,47 \\
\hline 14 - Quando me entregam uma avaliação, demoro um tempo para me acalmar e pensar em claridade. & 0,67 & 0,45 \\
\hline 13 - Não sou bom em realizar (responder) avaliações. & 0,63 & 0,40 \\
\hline 3 - Durante as avaliações eu penso muito nas consequências de uma reprovação. & 0,63 & 0,39 \\
\hline 11 - Quando eu faço uma avaliação difícil, me sinto reprovado mesmo antes de começar. & 0,63 & 0,39 \\
\hline 8 - Depois de realizar uma avaliação sinto que poderia ter feito melhor. & 0,61 & 0,38 \\
\hline 1 - A preocupação pelas avaliações me tira o sono. & 0,60 & 0,35 \\
\hline 9 - Realizar bem as avaliações me preocupa mais do que deveria. & 0,59 & 0,35 \\
\hline 12 - Nas avaliações, não demonstro tudo que sei acerca do tema. & 0,54 & 0,29 \\
\hline 15 - Nas avaliações não obtenho bons resultados. & 0,47 & 0,22 \\
\hline Número de itens & 16 & \\
\hline Valor próprio & 7,51 & \\
\hline Variância explicada (\%) & 46,94 & \\
\hline Alfa de Cronbach & 0,92 & \\
\hline Ômega de McDonald & 0,92 & \\
\hline
\end{tabular}

Estudo 2. Comprovação da Estrutura Fatorial da Cognitive Test Anxiety Scale (CTAS)

Considerando a estrutura unidimensional da CTAS, para os 16 itens validados no Estudo 1 , realizamos uma análise fatorial confirmatória (AFC), adotamos 0 método de estimação dos Mínimos Quadrados Ponderados Robustos (WLSMV), resultando nos seguintes indicadores de ajuste, descritos na Tabela 2: $\times 2 / \mathrm{gl}=1,63, \mathrm{CFI}=0,96, \mathrm{TLI}=0,95$, RMSEA $($ IC $90 \%)=0,06(0,04-0,07)$, Pclose $=$ 0,25 e SRMR $=0,05$. Ressalta-se que todos os valores específicos apresentaram lambdas $(\lambda)$ diferentes de zero $(\lambda \neq 0$; $F>3,84, p<0,05)$, como observado na Figura 1.
Como exposto na Figura 1, todos os pesos fatoriais indicam que os itens representam satisfatoriamente 0 construto, com cargas fatoriais $(\lambda)$ variando de 0,43 (item 15. Nas avaliações não obtenho bons resultados.) a 0,80 (item 4. Ao começar uma avaliação fico tão nervoso que muitas vezes não consigo pensar com clareza). A confiabilidade (precisão) da medida foi avaliada pelo alfa de Cronbach (considerando correlações policóricas) e pelo ômega de McDonald, apresentando valores iguais a 0,94. 


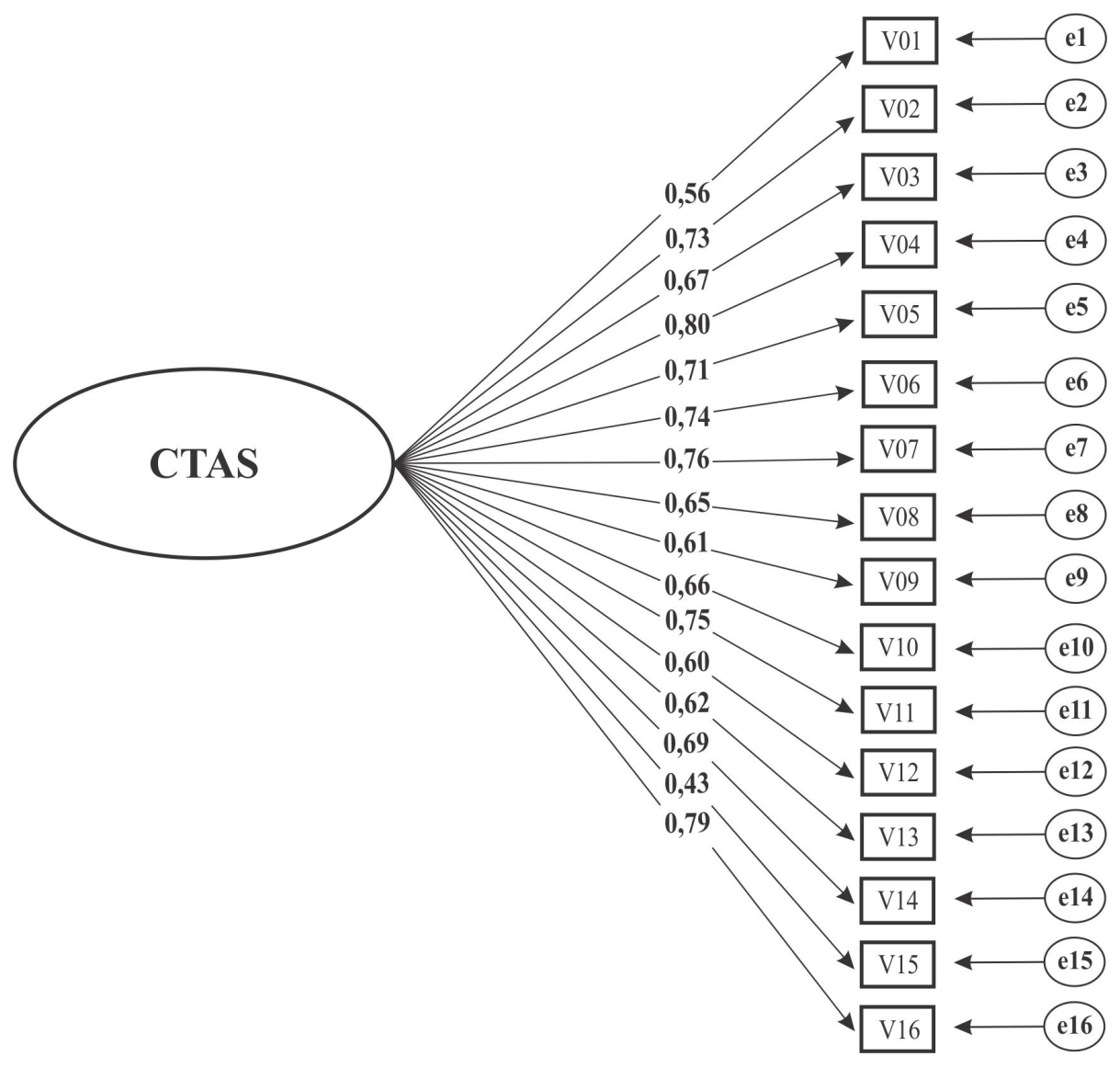

FIGURA 1.

Estrutura unifatorial da Cognitive Test Anxiety Scale.

\section{DISCUSSÃO}

A ansiedade frente a exames configura-se como um dos fenômenos mais recorrentes no contexto universitário (Ne'Eman-Haviv \& Bonny-Noach, 2019), percebe-se a necessidade de contar com medidas que possibilitem compreender adeaqudamente este fenômeno. Tendo em conta isto, 0 principal objetivo do estudo foi adaptar para o contexto brasileiro uma medida que avalie a ansiedade frente exames (Cassady \& Johnson, 2002; Furlan et al., 2009), além de conhecer evidências de validade fatorial e consistência interna da medida.

Reforça-se a importância desse empreendimento para que hajam avanços teóricos e metodológicos para o entendimento da AE no cenário nacional, pois contar com instrumentos com qualidades psicométricas adequadas e fundamentação teórica pertinente possibilitam hipotetizar, testar e compreender seus antecedentes e consequentes. Nessa direção, entendemos que a CTAS, configura-se com um dos instrumentos mais utilizados no cenário internacional, contribui para um melhor entendimento do fenômeno e seus correlatos. Dito isto, estima-se que os principais objetivos dessa pesquisa lograram êxito, pois 0 instrumento apresentou evidências consideráveis de qualidade métrica da CTAS.

Levando em consideração os principais achados da presente pesquisa, deve-se enfatizar que o Estudo 1, reuniu evidências 
que atestaram a qualidade métrica da medida (validade fatorial e precisão), pois apresentou índices superiores aos indicados pela literatura (Cohen, Swerdlik, \& Sturman, 2014; Pasquali; 2016; Tabachnick \& Fidell, 2013). Assim, para o contexto brasileiro, a CTAS ficou composta por 16 itens, que avaliam a ansiedade cognitiva frente as avaliações de forma global, com bom índice de precisão, avaliado pelos indicadores alfa do Cronbach e ômega de McDonald, este último considerado mais robusto, em detrimento do alfa de Cronbach (Dunn, Baguley, \& Brunsden,2014; McDonald, 1999).

Referente ao Estudo 2, que foi realizado, visamos reunir evidências psicométricas complementares da medida. Para tanto, consideramos análises fatoriais confirmatórias (AFC; Byrne 2011; Múthen \& Múthen, 2014), avaliamos o nível categórico(ordinal) do CTAS, por meio do estimador Weighted Least Squares Mean and Variance-Adjusted (WLSMV; Muthén \& Muthén, 2014).

Para tanto, por meio da estrutura unidimensional apontada no Estudo 1, foi possível observar que os dados empíricos se adequaram ao modelo teórico, previamente indicado (e.g., CFI e TLI>0,95 e RMSEA<0,08; Tabachnick \& Fidell, 2013), além de corroborar, a confiabilidade (precisão), que foi avaliada pelos indicadores de Alfa de Cronbach (matriz policórica) e 0 ômega de McDonald, com seus valores acima do ponto de corte indicado, ou seja, de no mínimo 0,70, sendo considedos meritórios (Cohen et al., 2014; McDonald, 1999). Os resultados apresentados corroboraram a estrutura unifatorial, já evidenciada em pesquisas transculturais, prévias (Baghaei \& Cassady, 2014; Cassady \& Finch, 2015; Furlan et al., 2009; Furlan et al., 2011; Turquia (Bozkurt et al., 2017).

Entretanto, cabe ressaltar que mesmo que os achados tenham sido promissores, faz-se necessário apontar as potenciais limitações da pesquisa. Nessa direção, podemos citar duas principais, a saber: questão amostral, que foi angariada de maneira conveniente (não probabilística), contando com a colaboração participantes que, convidados, aceitaram responder a pesquisa. Assim, o resultado deve ser considerado com reserva, pois existem diferenças em relação ao tamanho amostral quando considerados os diferentes cursos. Neste caso, deve-se ter em conta que estudantes de Psicologia representam mais de $50 \%$ da amostra angariada na presente pesquisa.

Tendo isto em conta, entende-se que os achados não permitem que os resultados reflitam a realidade para além da amostra considerada. Entretanto, ressalta-se que não se pretendeu generalizar resultados, mas apresentar uma medida de ansiedade frente a avaliações acadêmicas, que reunisse evidencias iniciais referente a validade baseada na estrutura interna da CTAS. Outra potencial limitação refere-se ao fato de terms aplicado uma medida de autorrelato (lápis e papel), que ocasiona algumas desvantagens, a exemplo da inerente a desejabilidade social, que faz com que 0 respondente possa alterar suas respostas, visando a autopromoção, pois sabe-se que que condutas ansiosas em contextos avaliativos não são desejáveis entre universitários (Ne'Eman-Haviv \& BonnyNoach, 2019).

Assim, considerando a possibilidade de serem realizadas pesquisas futuras, que utilizem a medida apresentada na presente pesquisa, indicamos algumas sugestões que tem por finalidade superar as potenciais limitações aqui encontradas. Dito isto, sugere-se que estudos futuros abranjam amostras maiores e mais heterogêneas, abrangendo um número maior de estados brasileiros, para verificar se a estrutura interna da CTAS se confirma. Disto isto, a validade relativa a estrutura interna, no processo de acumulo de evidências, é uma das primeiras a ser obtidas. Isto é justificável, pois conhecer a estretura do instrumento é 
condição para que se buquem evidências adicionais de validade, como por exemplo as baseadas em variáveis externas (convergente-discriminante e de critério) (Ambiel \& Carvalho, 2017). Assim, sugere-se relacionar com variáveis que possivelmente podem está associadas à ansiedade, tais como a procrastinação, autoestima e a traços de personalidade e depressão (Cassady et al., 2019).

Outroassim, seria igualmente interessante levar a cabo estudos que investiguem invariância fatorial, incluindo diferentes grupos (gênero, instituição pública/ particular, etc). Ademais, por meio da técnica DIF, seria possível verificar possíveis diferenças de respostas dos itens em função dos sexos dos participantes. Isso ajudaria a observar e esclarecer possíveis diferenças oriundas de características, manifestações ou aspectos da percepção ansiedade no exame em diferentes grupos (Bozkurt et al., 2017).

Ademais, seria importante diversificar as amostras, tendo em conta diferentes grupos de estudantes, devendo-se considerar diferentes níveis educacionais, a exemplo do ensino fundamental e médio, além de estudantes de pós-graduação (especialização, mestrado e doutorado), pois pode ser útil comparar participantes de diferentes faixas etárias que estão em distintos estágios do processo educativo (Donati et al., 2019).

Em suma, conclui-se que os resultados observados na presente pesquisa, indicam evidências da adequação da CTAS para 0 Brasil, que é uma mediada relativamente curta, composta por 16 itens, com boas qualidades psicométricas, que pode ser útil em pesquisas que visem estudar a ansiedade frente a exames (avaliações) e seus correlatos, possibilita avanços na temática no cenário nacional. Para além disso, os achados de pesquisas futuras podem subsidiar planos de intervenções ou politicas que visem reduzir a ansiedade cognitiva em universitários, ocasionando um maior bem-estar aos estudantes.

\section{REFERÊNCIAS}

Baghaei, P., \& Cassady, J. (2014). Validation of the Persian Translation of the Cognitive Test Anxiety Scale. SAGE Open, 4(4), 1-11. https://doi.org/10.1177/2158244014555 113

Borsa, J. C., Damásio, B. F., \& Bandeira, D. R. (2012). Adaptação e validação de instrumentos psicológicos entre culturas: algumas considerações. Paidéia, 22(53), 423-432. https://doi.org/10.1590/S0103863X2012000300014

Bozkurt, S., Ekitli, G. B., Thomas, C. L., \& Cassady, J. C. (2017). Validation of the Turkish Version of the Cognitive Test Anxiety Scale-Revised. SAGE Open, 7(1), 1-9.

https://doi.org/10.1177/2158244016669 $\underline{549}$

Byrne, B. (2010). Structural equation modeling with Amos: Basic concepts, applications and programming. Lawrence Erlbaum.

Cassady,. C. (2004). The influence of cognitive test anxiety across the learning testing cycle. Learning and Instruction, 14, 569-592. https://doi.org/10.1016/j.learninstruc.20 $\underline{04.09 .002}$

Cassady, J. C., Mohammed, A., \& Mathieu, L. (2004). Cross-cultural differences in test anxiety: Women in Kuwait and the United States. Journal of Cross-Cultural Psychology, 35, 715-718. https://doi.org/10.1177/0022022104270 $\underline{113}$

Cassady, J. C., \& Finch, W. H. (2015). Using factor mixture modeling to identify dimensions of cognitive test anxiety. Learning and Individual Differences. 41, 14-20.

https://doi.org/10.1016/j.lindif.2015.06.0 $\underline{02}$

Cassady, J. C., \& Johnson, R. E. (2002). Cognitive test anxiety and academic 
procrastination.

Contemporary

Educational Psychology 27(2), 270-295. https://doi.org/10.1006/ceps.2001.1094

Cassady, J. C., Pierson E. E., \& Starling. J. M. (2019). Predicting student depression with measures of general and academic anxieties. Frontiers in Education, $\quad 4(11), \quad 1-9$. https://doi.org/10.3389/feduc.2019.000 11

Cohen, R. J., Swerdlik, M. E., \& Sturman, E. D. (2014). Testagem e avaliação psicológica: Introdução a testes e medidas ( $8^{\circ} \mathrm{ed}$.). AMGH.

Dunn, T. J., Baguley, T., \& Brunsden, V. (2014). From alpha to omega: A practical solution to the pervasive problem of internal consistency estimation. British Journal of Psychology, 105(3), 399-412. https://doi.org/10.1111/bjop.12046

Donati, M. A., Izzo, V. A., Scabia, A., Boncompagni, J., \& Primi, C. (2019). Measuring test anxiety with an invariant measure across genders: The case of the German Test Anxiety Inventory. Psychological Reports, 123(4), 1-21. https://doi.org/10.1177/0033294119843 $\underline{224}$

Furlan, L.; Cassady, J.C. \& Pérez, E. (2009). Adapting the Cognitive Test Anxiety Scale for use with Argentinean university students. International Journal of Testing, 9(1), 3-19. https://doi.org/10.1080/1530505090273 3448

Furlan, L.; Pérez, E.; Moyano, M. \& Cassady, J.C. (2011). Propiedades psicométricas y estandarización de la Escala de Ansiedad Cognitiva frente a los exámenes a la población universitaria argentina. Evaluar, 10, 22-31. https://doi.org/10.35670/16674545.v10.n1.457

Furlan, Piemontesi, Heredia, \& Sánchez, (2015). Ansiedad ante los exámenes en estudiantes universitarios: Correlatos y tratamiento. Anuario de Investigaciones de la Facultad de Psicología, 2(1), 3753. https://revistas.unc.edu.ar/index.php/aif p/article/view/12629

Hoferichter, F., Raufelder, D., Ringeisen, T., Rohrmann, S., \& Bukowski, W. M. (2015). Assessing the multi-faceted nature of test anxiety among secondary school students: An English version of the German Test Anxiety Questionnaire: PAF-E. The Journal of Psychology, 150(4), 450-468.

https://doi.org/10.1080/00223980.2015. 1087374

Holgado-Tello, F. P., Chacón-Moscoso, S., Barbero-García, I., \& Vila-Abad, E. (2010). Polychoric versus Pearson correlations in exploratory and confirmatory factor analysis of ordinal variables. Quality \& Quantity, 44(1), 153-166.

https://doi.org/10.1007/s11135-0089190-y

Lara, S. A. D. (2014). ¿Matrices policóricas/tetracóricas 0 matrices Pearson? Un estudio metodológico. Revista Argentina de Ciencias del Comportamiento, 6(1), 39-48. http://www.redalyc.org/articulo.oa?id=3 33430869006

Liebert, R. M., \& Morris, L. W. (1967). Cognitive and emotional components of test anxiety: A distinction and some initial data. Psychological Reports, 20(3), 975-978. https://doi.org/10.2466/pr0.1967.20.3.9 $\underline{75}$

Lorenzo-Seva, U., Timmerman, M. E., \& Kiers, H. A. L. (2011). The Hull Method for selecting the number of common factors. Multivariate Behavioral Research, 46(2), 340-364. https://doi.org/10.1080/00273171.2011. 564527

Lorenzo-Seva, U., \& Ferrando, P. J. (2013). FACTOR 9.2: A comprehensive program for fitting exploratory and semiconfirmatory factor analysis and IRT models. Applied Psychological Measurement, 37(6), 497-498. https://doi.org/10.1177/0146621613487 $\underline{794}$ 
McDonald, R. P. (1999). Test Theory: A Unified Treatment. Erlbaum.

Muthén, L. K., \& Muthén, B. O. (2014). Mplus user's guide. (7th Ed.). Muthén \& Muthén.

Ne'Eman-Haviv, V., \& Bonny-Noach, $\mathrm{H}$. (2019). Substances as self-treatment for cognitive test anxiety among undergraduate students. Journal of Psychoactive Drugs, 51(1), 78-84. https://doi.org/10.1080/02791072.2018. 1564090.

Pasquali, L. (2016). TEP - Técnicas de Exame Psicológico: Os Fundamentos $\left(2^{\mathrm{a}} \mathrm{ed}\right)$. Vetor editora.

Piemontesi, S. E., \& Heredia, D. E. (2009). Afrontamiento ante exámenes: desarrollo de los principales modelos teóricos para su definición y medición. Anales de Psicología, 25(1), 102-111. http://www.redalyc.org/pdf/167/167115 94012.pdf

Putwain, D. W., Daly, A. L., Chamberlain, S., \& Sadreddini, S. (2015). Academically buoyant students are less anxious about and perform better in high-stakes examinations. British Journal of Educational Psychology, 85(3), 247263. https://doi.org/10.1111/bjep.12068

Revelle, W. (2013) Psych: Procedures for Personality and Psychological Research. Northwestern University, Evanston. $\quad$ http://CRAN.Rproject.org/package=psych

Rossel, Y. (2012). Lavaan: na R package for structural equation modeling. Jounal of Statistical Sofware, 48(2), 1-36. https://doi.org/10.18637/iss.v048.i02

Sánchez-Rosas, J., \& Furlan, L. A. (2017). Achievement emotions and achievement goals in support of the convergent, divergent and criterion validity of the Spanish-Cognitive Test Anxiety Scale. International Journal of Educational Psychology, 6(1), 67-92. https://doi.org/10.17583/ijep.2017.2286

Sarason, S. B., \& Mandler, G. (1952). Some correlates of test anxiety. Journal of Consulting and Clinical Psychology, 47(4), 810-817.

https://doi.org/10.1037/h0060009

Sarason, I. G. (1984). Stress, anxiety, and cognitive interference: Reactions to tests. Journal of Personality and Social Psychology, 46(4), 929-938. https://doi.org/10.1037/00223514.46.4.929

Spielberger, C.D. (1980) Test Anxiety Inventory. Consulting Psychologists Press.

Tabachnick, B. G., \& Fidell, L. S. (2013). Using multivariate statistics (6th ed.).Pearson Education.

Thomas, C. L., Cassady, J. C., \& Heller, M. L. (2017). The influence of emotional intelligence, cognitive test anxiety, and coping strategies on undergraduate academic performance. Learning and Individual Differences, 55, 40-48. http://dx.doi.org/10.1016/j.lindif.2017.03 $\underline{.001}$

Todos los trabajos publicados en Revista Salud \& Sociedad (ISSNe:0718-7475) están sujetos a una licencia Creative Commons Reconocimiento 4.0 Internacional

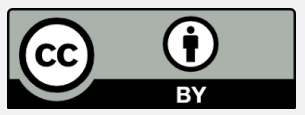




\section{RESUMO}

ANTECEDENTES: A ansiedade cognitiva refere-se a preocupações excessivas, antes, durante e depois das avaliações, interferindo no desempenho e ocasionando o fracasso acadêmico, além de estar associada positivamente o com neuroticismo e a depressão. OBJETIVO: Objetivou-se reunir evidências de qualidade psicométrica da Cognitive Test Anxiety Scale (CTAS) no Brasil. MÉTODO: Foram realizados dois estudos: No estudo 1 participaram 210 universitários da cidade de Parnaíba, Piaú, Brasil $\left(M_{\text {idade }}=21,7 ; \mathrm{DP}=3,73\right)$, a maioria cursando Psicologia (60\%) e mulheres (58,6\%).Responderam a CTAS e questões demográficas. RESULTADOS: A análise fatorial exploratória sugeriu uma solução unidimensional, que explicou $46,94 \%$ da variância total dos itens. A confiabilidade foi avaliada pelos indicadores alfa de Cronbach $(\alpha)$ e ômega de $\operatorname{McDonald}(\omega)$ iguais a 0,92. № Estudo 2, participaram 274 universitários de diferentes regiões brasileiras (Midade $=21,7 ; \mathrm{DP}=3,73$ ), a maioria do estado do Piauí $(60 \%)$, cursando Psicologia $(60,4 \%)$. Uma análise fatorial confirmatória apontou indicadores adequados ( $C F I=0,96 ; T L I=0,95 ; R M S E A=0,06)$. A confiabilidade foi satisfatória: $(\alpha)$ e $(\omega)$ iguais a 0,94. CONCLUSÃO: Foram encontradas evidências psicométricas para 0 Brasil, podendo a CTAS ser útil para interessados em investigar a temática e seus correlatos.

PALAVRAS-CHAVE: Ansiedade Frente a Teste, Estudantes, validade, análise fatorial.

\section{RESUMEN}

ANTECEDENTES: La ansiedad cognitiva se refiere a preocupaciones excesivas antes, durante y después de exámenes, que interfieren con el rendimiento, provocan fracaso académico, y se asocia positivamente con el neuroticismo y la depresión. OBJETIV0: El objetivo fue evaluar las propiedades psicométricas del Cognitive Test Anxiety Scale (CTAS) en Brasil. Método: Realizamos dos estudios: en el Estudio 1, participaron 210 estudiantes universitarios de la ciudad de Parnaíba, Piauí, Brasil (Medad $=21.7$; SD=3.73), la mayoría de ellos estudiantes de Psicología (60\%) y mujeres (58.6\%). Respondieron el CTAS y a preguntas demográficas. RESULTADOS: El análisis factorial exploratorio sugiere una solución unidimensional, que explica el $46.94 \%$ de la varianza total de los ítems. La fiabilidad se evaluó mediante los indicadores alfa de Cronbach $(\alpha)$ y omega de McDonald $(\omega)$ iguales a 0.92 . En el Estudio 2, participaron 274 estudiantes universitarios de diferentes regiones brasileñas $(M e d a d=21.7 ; S D=3.73)$, la mayoría del estado de Piauí (60\%), estudiando Psicología (60.4\%). Un análisis factorial confirmatorio apuntó indicadores adecuados ( $\mathrm{CFI}=0.96 ; \mathrm{TLI}=0.95 ; \mathrm{RMSEA}=0.06)$. La fiabilidad fue satisfactoria: $(\alpha)$ y $(\omega)$ iguales a 0,94. CONCLUSIÓN: Encontramos evidencia psicométrica para Brasil, que indica que el CTAS puede ser útil para aquellos interesados en investigar el tema y sus correlatos.

PALABRAS CLAVE: Ansiedad ante Pruebas, Estudiantes, validación, análisis factorial. 